\title{
Mejorando una relación terapéutica hostil con la psicoterapia analítica funcional
}

\author{
Lourdes Ascanio Velasco \\ Rafael Ferro García \\ María Rodríguez Bocanegra \\ Centro de Psicología Clínica C.E.D.I. Granada
}

\section{ResUmen}

La Psicoterapia Analítica Funcional (FAP, Kohlenberg \& Tsai, 1991) es un poderoso tratamiento en sí mismo y también una aproximación integradora con cualquier terapia produciendo unos resultados sinérgicos. Enfatiza el uso de la relación terapéutica para producir el cambio en los clientes, centrándose en las contingencias directas y naturales que ocurren en sesión. Es una terapia contextual que ha demostrado ser útil para el tratamiento de personas que presentan problemas de larga duración, difusos en su definición, vinculados a las dificultades en las relaciones de intimidad. Este trabajo expone la mejora de una relación terapéutica hostil a través de FAP. Se presenta un caso de una joven con discapacidad intelectual leve y con una historia de maltrato sospechado y abandono familiar, que presentaba dificultades en las relaciones de intimidad. Los resultados de esta intervención indicaron una mejoría en las relaciones terapéuticas que permitieron trabajar con más facilidad otras áreas de la vida de la cliente.

\section{PaLAVRAs-ChaVe}

Psicoterapia Analítica Funcional, Relaciones Terapéuticas Hostiles, Caso Clínico.

\section{ABSTRACT}

Functional Analytic Psychotherapy (FAP, Kohlenberg \& Tsai, 1991) is a powerful treatment itself and also an integrative approach with any therapy producing synergistic results. It emphasizes the use of the therapeutic relationship to bring about change in clients by focusing on direct and natural contingencies that occur in session. This contextual therapy has proven to be useful for treating people who have long-term problems, vague in its definition, related to difficulties in relationships of intimacy. This paper exposed the improvement of a hostile therapeutic relationship through FAP. A case of a young woman with mild intellectual disability and a history of suspected mistreatment and family abandonment, which presented difficulties in intimate relationships, is presented. The results of this intervention indicate an improvement in the therapeutic relationships which can be generalized to other areas of the life of the client.

\section{KeYWORDS}

Functional Analytic Psychotherapy, Hostile Therapeutic Relationship, Clinical Case. 


\section{Introducción}

La Psicoterapia Analítica Funcional (FAP, Kohlenberg \& Tsai, 1991) es una terapia que surgió en la década de los 90, y está considerada dentro de las llamadas terapias contextuales (Pérez Álvarez, 2014). FAP es un poderoso tratamiento en sí mismo y también una aproximación integradora con cualquier terapia produciendo unos resultados sinérgicos (Kohlenberg et al., 2005). Enfatiza el uso de la relación terapéutica para producir el cambio en los clientes, centrándose en las contingencias directas y naturales que ocurren en sesión, lo cual supone un alto grado de implicación por parte del terapeuta. Es una terapia que ha demostrado ser útil para el tratamiento de personas que presentan problemas de larga duración, difusos en su definición, vinculados a las dificultades en las relaciones de intimidad, que producen aislamiento social, hostilidad, fobia social, etc. (Martín-Murcia \& Ferro-García, 2015).

\section{Fundamentos de FAP}

FAP se fundamenta en el análisis funcional de lo que ocurre en sesión, en las contingencias de reforzamiento que se dan en el contexto de las relaciones terapéuticas y en la equivalencia funcional entre el ambiente clínico y el diario del cliente (Valero \& Ferro, 2015). Se proponen tres tipos de conductas clínicamente relevantes (CCR). Las CCR tipo 1 son los problemas del cliente en sesión; las CCR 2 son las mejoras del cliente en sesión; y las CCR 3, son las descripciones que hace el cliente sobre su propia conducta y los estímulos elicitadores, discriminativos y reforzantes que están asocia- dos a ella. Se proponen también cinco reglas terapéuticas que el terapeuta debe tener en cuenta durante las sesiones clínicas y que ayudan a los procesos de cambio: 1) observar las CCR, 2) evocarlas, 3) reforzar las CCR 2,4) ver el efecto de la conducta del terapeuta sobre las CCR y 5) generar un repertorio de descripción de las relaciones funcionales entre las variables de control y la conducta (Kohlenberg \& Tsai, 1991; Tsai, Kohlenberg, Kanter, \& Waltz, 2009).

\section{Teoría del desarrollo del yo}

Una de las aportaciones más importantes de FAP es su teoría sobre el desarrollo del yo, de sus patologías y su tratamiento. El desarrollo del yo se realiza a través de un entrenamiento social y verbal que realizan los cuidadores del niño, y la clave del mismo radica en transferir el control de las respuestas "yo X", de la estimulación pública a la privada. Es decir, poner el yo bajo control de la propia estimulación privada (sensaciones, emociones o necesidades). Del mismo modo, se puede establecer un desarrollo desadaptativo quedando bajo control público algunas o todas estas relaciones verbales del yo. En función del tipo de relación afectada, se puede presentar un yo inseguro si no se sabe qué se siente, se quiere o se piensa. Cuando las opiniones de uno están bajo control de los demás y se es muy sensible a las críticas, se puede mostrar un yo inestable. Las consecuencias de este problema son claras: una dependencia de los demás, sentirse vacío cuando se está sólo, no saber quién se es, evitar las relaciones de intimidad porque son aversivas e imprevisibles, etc. En ningún caso se trata de problemas de asertividad. Se- 
gún Valero y Ferro (2015), estos trastornos del yo son muy frecuentes en la clínica y pueden ser considerados factores trans-diagnósticos que recorren de forma transversal muchos de los problemas psicológicos que se definen en los sistemas de clasificación DSM o CIE. Además, la Teoría del Marco Relacional (Dymond \& Roche, 2013; Hayes, Barnes-Holmes, \& Roche, 2001) complementaría esta teoría de la formación del yo y explicaría la toma de perspectiva y la empatía. Según esta teoría habría tres marcos relacionales llamados deícticos, claves para este entrenamiento. Los marcos interpersonales (yo-tú), los marcos espaciales (aquí-allí) y los temporales (ahora-antes). Cuando hablamos con otra persona lo hacemos desde la perspectiva del yo localizado aquí-ahora sobre eventos que han ocurrido allí-entonces. La falta de empatía es un problema del marco interpersonal yo-tú y que tiene que ver con la toma de perspectiva, también. Desde FAP los hechos traumáticos conllevan graves problemas en las relaciones personales de intimidad y en la formación del yo, por lo que esta terapia es una buena alternativa para el tratamiento de estas secuelas del trauma.

\section{Habilidades terapéuticas desde FAP}

En FAP se parte de que muchos de los problemas psicológicos están relacionados con las relaciones de intimidad, y también de que establecer una relación intensa y curativa, además de ser un objetivo en terapia, está relacionado con la solución de tales problemas. Para crear este tipo de relaciones se debe tener en cuenta una serie de habilidades terapéuticas. Valero y Ferro (2017) han expuesto por un lado, unas habilidades personales como unas competencias o destrezas que van a permitir establecer esas relaciones de intimidad y por otro lado, unas habilidades clínicas más teóricas que están relacionadas con las reglas de la propia FAP.

Antes que nada hay que aclarar que el agente de cambio no son las habilidades terapéuticas sino los procesos conductuales que se dan en la interacción, y esas habilidades solo son la forma de aplicar mejor las técnicas (Valero \& Ferro, 2015).

Las habilidades personales deben adquirirse a través de la práctica como terapeuta y/o la supervisión, son fruto de las contingencias y serían: ser afectivo y cálido en la relación, generar confianza, ser auténtico y tener empatía. Además, para poder hacer esto, hay que conocerse a sí mismo y conocer qué problemas puede tener el terapeuta en sus relaciones sociales y personales que pudieran afectar en su trabajo.

Las habilidades clínicas pueden resumirse en el subtítulo del segundo manual de la terapia (Tsai et al., 2009): conciencia (Regla 1 y 4), coraje o valentía (Regla 2), amor (regla 3) y conductismo. Tener conciencia de lo que ocurre en sesión significa por un lado, observar las posibles CCR del cliente y por otro, ver los efectos que tiene la conducta del terapeuta sobre la del cliente y las propias reacciones del terapeuta ante las CCR como indicadores de lo que pueden sentir los demás en situaciones equivalentes. Una forma de ser conscientes de las posibles CCR es usar una conceptualización del caso, como se verá más adelante. Tener coraje o ser valientes durante la terapia se re- 
fiere a no tener miedo para evocar las CCR1 y que esto sea una oportunidad para desarrollar CCR2. Las CCR son idiosincráticas porque pertenecen a una historia única del cliente y del mismo modo la relación debe ser única para esa persona. Creando como se ha dicho una relación íntima y de confianza mutua, siendo auténticos y dando y recibiendo afecto, el terapeuta en este contexto debe asumir riesgos (Valero \& Ferro, 2017), en este sentido se dice que hay que ser valientes. Además, el término "amor terapéutico", introducido por Tsai et al. (2009), implica un interés auténtico y genuino por el cliente, sin referirse a unos sentimientos de "amor" en el sentido social habitual. Valero y Ferro (2015) definen "amor terapéutico" como aquello que implica cariño, benevolencia, sensibilidad, compasión, ternura, y comprensión hacia todo lo relacionado con el cliente. Buscando el beneficio para este último, el terapeuta debe ser atento y afectuoso de manera incondicional, de forma similar a una relación de intimidad en la vida cotidiana, y reforzar las mejorías de forma natural.

A continuación, se expone la intervención desde FAP en el caso de una joven con discapacidad intelectual leve, con una sospecha de historia de maltrato y con un abandono familiar, que presentaba dificultades en las relaciones de intimidad generadas por un problema del yo y una falta de empatía. Actualmente la cliente continúa en tratamiento psicológico, validando su yo y mejorando su lenguaje y su lecto-escritura. La exposición de esta intervención pretende exponer las habilidades terapéuticas desde FAP en un caso difícil para mejorar las relaciones terapéuticas y de intimidad en la vida diaria. Existe a día de hoy una escasa bibliografía de esta problemática.

\section{Método \\ Descripción del caso clínico}

Teresa tenía 24 años cuando acudió a consulta. Era la mayor de dos hermanas. Según los criterios diagnósticos del DSM-5 (APA, 2013), Teresa tiene una discapacidad intelectual leve (F70) con limitaciones en el comportamiento adaptativo en los dominios conceptual, social y práctico. Sufrió abandono por parte de sus padres biológicos y maltrato físico y psicológico sospechados en la institución de acogida hasta los 8 años de edad, cuando fue adoptada junto a su hermana por su actual familia. En el momento de la adopción, su lenguaje no era comprensible y necesitaba la ayuda de su hermana para hacerse entender. Al acudir a consulta también cumplía los criterios, según el DSM-5, del trastorno del lenguaje (F80.2), con dificultades en la adquisición y uso del lenguaje (hablado y escrito). Entre los errores al hablar, no pronunciaba algunos sonidos y con una alta frecuencia omitía los artículos determinados en las frases, no utilizaba autoclíticos descriptivos (Skinner, 1957) y su velocidad de habla era alta, por lo que el oyente a menudo era incapaz de entender lo que decía. Presentaba dificultades de lectura, cometía errores leves (vacilaciones, repeticiones y rectificaciones) y omitía palabras, y tenía dificultades en la expresión escrita, con errores gramaticales y de contenido. Los padres comentaron que Teresa tenía ataques de ira, baja resistencia a la frustración y no aceptaba las correcciones de los demás. Tenía un estilo de atribución exter- 
na de los hechos, achacando a otras personas la causa de sus problemas. Se mostraba desconfiada con los demás. Tenía conflictos familiares, especialmente con su hermana y con su abuela, con las que discutía con frecuencia. Era rencorosa y los enfados le duraban varios días. Entre la primera y la segunda sesión, llegó a agredir a su madre por negarse a darle algo que ella quería. Había acudido a pedir ayuda a otros centros de psicología, de logopedia, hospitales, clases particulares, etc., sin conseguir mejoras. Las demandas de los padres en consulta fueron: que Teresa se comportara respetuosamente y especialmente con su hermana y con su abuela, que aprendiera a dominar su ira, aceptara sus errores, y mejorara las relaciones con los demás. Además, pidieron ayuda para mejorar el lenguaje, la lectura, la escritura y la orientación temporal de Teresa, ya que no sabía el uso del reloj, calcular las horas, usar el calendario, etc. Aunque la demanda fue generada por los padres, Teresa acudió a consulta de manera voluntaria.

\section{Análisis y conceptualización del caso}

Como una guía para la terapeuta para responder de manera eficaz a las CCR del cliente, se llevó a cabo una forma de conceptualización del caso clínico (Kanter, Weeks, Bonow, Landes, Callaghan, \& Follette, 2009; Kohlenberg, Kanter, Bolling, Parker, \& Tsai, 2002; Kohlenberg y Tsai, 2000; Valero \& Ferro, 2015). Un registro abierto, donde se describen variables que se suponen claves para el análisis de un caso clínico (Ferro, Valero, \& López, 2009):

Problemas en la vida diaria. Son los problemas que el cliente presenta en su vida diaria, y que la terapeuta propone. Teresa se mostraba hostil y verbalmente agresiva en sus relaciones de intimidad, especialmente con su abuela y con su hermana. Tenía explosiones de ira intermitentes de alta intensidad. Tendía a hacer atribuciones externas de sus problemas: culpaba a los demás de las cosas desagradables que le ocurrían o se quejaba si algo le ocurría a ella y no a los demás. Respondía verbalmente de manera literal y no entendía ironías, ni bromas. No era empática con las personas que le rodeaban. Su lenguaje era a menudo incomprensible y no aceptaba las correcciones. La familia mantenía los problemas de conducta, atendiendo a Teresa ante un mal comportamiento y/o cediendo si lo consideraban oportuno para no incrementar sus enfados.

Historia relevante. Se trata de las variables históricas que pueden ayudar a comprender la conducta actual. Teresa había sido abandonada junto a su hermana por sus padres biológicos. Fue institucionalizada en un centro de acogida en el que se sospechó que recibió maltrato psicológico y físico. Fue adoptada junto a su hermana a los 8 años por una misma familia. A los 8 años necesitaba ayudas de su hermana para ser entendida por los demás. Cumplía los criterios de una discapacidad intelectual leve y un trastorno del lenguaje. Acudió a diferentes profesionales sin conseguir mejoras. Presentaba un posible problema del yo inseguro/inestable.

Problemas en sesión (CCR1). Son los problemas del cliente que se dan en sesión, equivalentes funcionalmente a los que ocurren fuera de la terapia. Teresa no mostraba una actitud colaboradora en la sesión clínica. Se mostraba 
hostil con la terapeuta. Se mostraba desconfiada. No sabía qué pensaba ni qué quería. Utilizaba la evitación y el escape cuando no quería hablar de algún tema (por ejemplo, de su pasado) o hacer algo (por ejemplo, leer). Se quejaba de los demás. Presentaba frecuentes mandos disfrazados (Kohlenberg \& Tsai, 1991) en forma de críticas hacia los demás y/o hacia la propia terapeuta, como una manera de pedir o eliminar algo. Era impaciente (se enfadaba si la terapeuta no era puntual). No aceptaba el fracaso (se enfadaba si perdía en el juego).

Conceptos cognitivos. Son las interpretaciones, creencias, asunciones erróneas que la cliente hace de lo que le ocurre y de lo que le ha pasado, y también sobre la salud, su bienestar psicológico, etc., que pueden interferir en su tratamiento. Teresa relacionaba su hostilidad como algo genético ("Es que tengo un gen", refiriéndose a que tenía un gen que la hacía comportarse de manera hostil). Para ella era equivalente tener personalidad con tener mal carácter. Pensaba que los demás eran responsables de sus problemas. Tenía problemas del yo ("No sé cómo soy yo, nunca lo he pensado...").

Objetivos en la vida diaria. Es decir, lo que se pretende conseguir y generalizar a la vida de esa persona. Se planteó como objetivos que mejorara la relación con su familia, y especialmente, con su abuela y su hermana. También que aceptara las correcciones. Que se mostrara cordial en sus relaciones íntimas y sociales. Que hiciera mandos de manera adecuada. Que mejorara su topografía vocal. Que mejorara su lectura y escritura.

Objetivos en sesión (CCR2). Estos son los objetivos que se marca la terapeuta en la propia sesión. Se plantearon que pusiera bajo control privado sus respuestas "yo X". Que hablara de cómo es ella. Que hablara de su recuerdo del pasado. Que hiciera mandos de manera adecuada. Que estableciera relaciones funcionales correctas (CCR3). Que eliminara las quejas hacia los demás. Que aceptara esperar (por ejemplo, en la sala de espera antes de entrar a sesión). Que aceptara el fracaso (por ejemplo, perder en el juego). Que aceptara las correcciones (del lenguaje, lectura y escritura).

\section{Intervención}

La intervención que aquí se describe, se refiere al período comprendido entre las sesiones 1 a la 12, con una duración de 6 meses. Los datos de la historia de Teresa recogidos durante la entrevista a los padres fueron fundamentales, pues Teresa evitaba responder a las preguntas de la terapeuta desde el principio de la intervención.

\section{Fase Inicial}

A la $1^{\text {a }}$ sesión acudieron únicamente los padres. Se recogieron datos sobre su historia y se marcaron objetivos con ellos.

Teresa acudió a consulta por primera vez a la $2^{\text {a }}$ sesión. Durante la entrevista hubo una frecuencia de CCR1 muy elevada, siendo su actitud no colaboradora: no respondía a preguntas abiertas e intentaba controlar ella la sesión. Se hipotetizó que era una equivalencia funcional de lo que ocurría en su casa (los padres decían que era una "mandona"). Cuando la terapeuta le pidió que le hablara sobre ella, Teresa se cruzó de brazos y contestó: "Tú dime y yo te contesto". Contestaba de un modo hui- 
dizo, dando respuestas ambiguas. Cuando se le preguntó si sabía por qué venía a consulta, contestó: "no lo sé, dímelo tú, que para eso eres psicóloga". Otras veces respondía con silencios o con risas histriónicas. Estas conductas se interpretaron como conductas de escape o evitación. Únicamente respondía a través de monosílabos a preguntas cerradas. Aparecieron problemas cuando se jugó a un juego de mesa: cuando iba perdiendo en éste, Teresa golpeó la mesa y la mano de la terapeuta con la función de evitar que ésta le ganara. No se corrigió el lenguaje en ninguna ocasión, intentando ser una audiencia no punitiva (Skinner, 1953).

En la $3^{a}$ sesión, se continuó recogiendo datos. Se intentó corregir una palabra a través de imitación vocal, pero se negó y no se insistió en ese momento, aunque más adelante se volvió a presentar el estímulo, para no reforzar su negativa. Aparecieron mandos disfrazados con una alta frecuencia (CCR1). Espontáneamente comentó que le gustaba hacer muñecos de papel y se le preguntó qué muñecos sabía hacer. Teresa siguió insistiendo en este comentario pero no ponía ejemplos de lo que sabía hacer (CCR1), se hipotetizó su cometario como un posible mando disfrazado (regla 1) y la terapeuta dejó de responder a ese comentario, intentando extinguirla. Se le evocó un mando directo diciéndole "Oye, si quieres hacer alguno, no tienes más que pedirme folios y yo te los doy" (regla 2), lo que provocó una petición directa en la cliente (CCR2) que fue reforzada dándole inmediatamente los folios, comportándose de esta forma natural, contingente y auténtica. También dijo de forma espontánea:"Yo soy muy lista" (CCR2, una respuesta "yo X"), después de realizar un muñeco de papel. Cuando la terapeuta le hacía preguntas para obtener información sobre su historia, la cliente resoplaba o se quedaba en silencio sin contestar (CCR1). En cierto momento la terapeuta dijo: "pídeme que deje de hacerte preguntas si es lo que quieres, por favor" (regla 2), modelando cómo llevar a cabo un mando directo, pero Teresa continuó resoplando. En este caso, se aplicó un reforzamiento diferencial respecto al ejemplo de la demanda de folios, es decir, cuando volvió a resoplar, no se atendió a éste y la terapeuta continuó preguntando. Se utilizaron juegos sencillos de palabras con alta probabilidad de éxito (regla 2), como decir palabras que comenzaran por una letra determinada o decir frases que contuvieran una palabra en concreto, para intentar que aceptara las correcciones. Al final de la sesión se jugó de nuevo a un juego y ganó la terapeuta. Esta vez se le pidió a Teresa directamente que felicitara a la persona que había ganado y así lo hizo (CCR2).

A la 4a sesión, llegó a consulta muy nerviosa y tanto ella como su familia relacionaban este estado a que tenía un examen en pocos días. Cuando la terapeuta intentó corregir su lenguaje, ya que no entendía lo que decía, aparecieron quejas, negativas y amenazas (CCR1), que fueron todas extinguidas. La extinción consistía en no responder a sus comentarios y continuar con las correcciones. Al final de la sesión se dedicaron unos minutos a hacer ejercicios sencillos de conducta conceptual: decir series de números de 2 en 2, nombrar frutas, decir los días de la semana y los meses, etc., aplicando reforzamiento diferencial dependiendo de que intentara corregirse o se negara. 
En la $5^{\text {a }}$ sesión Teresa se mostró enfadada por, según ella, haberle hecho trabajar demasiado durante la sesión anterior. La terapeuta hipotetizó esta queja como un mando disfrazado (CCR1) para no trabajar el lenguaje durante esta sesión. Aparecieron comentarios del tipo "no te hagas la tonta", "no te creo", "si hay mucho que hacer me iré escupida de aquí" (CCR1), que eran extinguidos. Como se hipotetizó que la forma de trabajar el lenguaje durante la sesión 4 fue muy aversiva para ella, se decidió trabajarlo de forma espontánea, aprovechando esta vez conversaciones que surgían entre terapeuta y paciente. Se incrementó su colaboración y comenzó a hacer preguntas personales a la terapeuta (CCR2). Se usó la respuesta a esas preguntas personales, es decir, las auto-revelaciones de la terapeuta como una consecuencia natural que mejoraría la intimidad, tal y como proponen Tsai et al. (2010). Como en las sesiones anteriores habían aparecido CCR1 al jugar a juegos de mesa, y se hipotetizó que la cliente estaba intentando evitar perder, se volvió a presentar el juego al final de esta sesión. Teresa volvió a palmear la mano de la terapeuta y lanzó una ficha al aire (CCR1) cuando ésta última iba ganando en el juego. A pesar de su conducta, se le pidió continuar la partida con la intención de bloquear la evitación.

\section{Fase Intermedia}

En FAP no hay una delimitación clara entre una fase y otra, depende del cliente y su problemática (Valero \& Ferro, 2015), se hipotetizó que hubo un cambio en Teresa en cuanto a su resistencia al cambio y una mejora en la relación con la terapeuta, a partir de la sesión clíni- ca anterior. Durante la $6^{\text {a }}$ sesión Teresa mostró una actitud más dispuesta a trabajar (CCR2), aceptó las correcciones del lenguaje (CCR2) y también imitó palabras y frases de mayor complejidad que en las sesiones anteriores. Apareció la primera aproximación a hablar de su pasado (CCR2). Cuando ella dejó de hablar de éste, porque según dijo "le traía malos recuerdos" (regla 5), no se le insistió y se elogió su confianza al contarlo, sin profundizar en el contenido de lo que había contado para no castigar. Se consideró una mejoría el hecho de que fuera capaz por primera vez de interpretar qué le ocurría (CCR3) y dar una respuesta "yo X", cuando habló de su pasado diciendo que le venían malos recuerdos y se sentía mal.

Durante la 7a sesión, la madre de Teresa informó a la terapeuta que no había agredido verbalmente a su hermana ni a su abuela desde que venía a consulta, aunque continuaba respondiéndoles mal cada vez que éstos le hacían una crítica o le corregían. La madre se quejó de su falta de empatía. Aunque no percibía mejoras en el lenguaje, comentó que la notaba más motivada en mejorarlo y que a veces comentaba en voz alta "cada vez hablo mejor". Cuando 30 minutos después Teresa entró en sesión presentaba resistencia a hablar y respondía a las preguntas de modo huidizo (CCR1). Estas CCR podrían tener causación múltiple, como que entrara la madre antes que ella y que tuviera que esperar 30 minutos en la sala de espera. En este contexto, se decidió que había que trabajar la empatía intercambiando los marcos relacionales deícticos interpersonales: yo-tú (Barnes-Holmes, Hayes, \& Dymond, 2001; Kohlenger, Tsai, Kanter, \& Parker, 2009; 
McHugh, Stewart, \& Hooper, 2012). En un primer momento, se le pidió que se pusiera en el lugar de la terapeuta preguntándole cómo se sentiría si ésta no respondiera a sus preguntas. No hubo respuesta por parte de ella (CCR1). Se le preguntó qué le gustaría hacer. Pidió escribir en un papel cómo era su hermana y cómo era ella (CCR2), y se reforzó la respuesta dándole un lápiz y una hoja de papel e interactuando con ella. De esta manera, se pretendía reforzar de forma diferencial respecto a la situación anterior. Tras comentar las disputas que tenía con su hermana, se trabajó en solución de conflictos como una manera de reforzar la colaboración y los "yo X". Se le propuso que durante las próximas semanas describiera a la hermana todo lo que hiciera que le fuera agradable, pero no con la intención de que siguiera las instrucciones, sino como una consecuencia natural a su cooperación.

En la $8^{a}$ sesión se comprobó que había seguido las instrucciones (CCR2) sobre la solución de conflictos, así, podríamos decir que la consecuencia natural de dar instrucciones a una demanda de los conflictos con su hermana había promocionado la generalización (regla 4). Aunque continuó quejándose de ella (CCR1).

Se intentó valorar qué objetivos y metas se planteaba:

Terapeuta (T): “Qué cambiarías de ti?"

Cliente (C): "Cambiaría a mi hermana" (CCR1).

T: "¿Pero qué cambiarías de ti misma?" Se bloqueó la evitación de hablar sobre ella misma y se extinguió la crítica a la hermana.

C: "Eso no lo he pensado nunca... ese puede ser el problema. Estoy en blanco."
T: "¿Qué te pasa por la cabeza en este momento?"

\section{C:"Pienso en mi hermana..."}

Ante la duda de que fuera un escape, se ignoró esta respuesta, pero se volvió al tema más adelante, sin conseguir que se hablara de lo que tendría que cambiar. Se comprobó que la terapeuta no reforzó la respuesta anterior. A continuación, para romper esta situación, se intentó moldear su respuesta, dándole un tipo de ayuda, tal y como proponen Kohlenberg y Tsai (1991):

T: “Qué cambiarías de ti, físicamente?"

C: "Nada, yo estoy bien asi"

La ayuda de hablar de algo físico no funcionó.

T: "¿Y de tu forma de ser?"

C: "Cambiaría mi carácter" (CCR2)

T: "Ah, cambiar tu carácter! Vaya...!" Y no se volvió a insistir.

Al final de la sesión se trabajaron series numéricas, conceptos verbales y el lenguaje. Aceptó llevar a cabo las actividades y ser corregida (CCR2). Para trabajar sobre esta última área se continuó utilizando un lenguaje espontáneo. Al final de la sesión, y como parte de una conversación espontánea sobre los libros que tenía en casa, la terapeuta le pidió a Teresa que le escribiera por correo electrónico el nombre de algunos de ellos, intentado generar un compromiso con la terapia fuera de la sesión. De nuevo la madre indicó que la cliente se había mostrado más cariñosa con la hermana y su relación había mejorado durante las últimas semanas.

Parece que Durante la sesión 9a la cliente dijo estar controlando su reacción cuando se enfadada con su hermana y con su abuela (CCR2). Hizo 
comentarios del tipo"es que mi abuela la pobreya es mayor..."o"mi hermana se porta como una hermana" poniéndose en el lugar de éstas (CCR2). Se reforzaron este tipo de descripciones, asintiendo y siguiendo atentamente la conversación (regla 3). Por primera vez en consulta se atribuyó parte de responsabilidad al contar un problema que había tenido con su hermana, diciendo "reconozco que yo también le sigo las bromas", refiriéndose a que se chinchaban mutuamente (CCR2). Se trabajaron tareas de lectura (leer un texto corto) y escritura (escribir palabras) por primera vez y sin recibir negativas (CCR2).

Antes de comenzar la 10a sesión, Teresa y su madre tuvieron que esperar 20 minutos en la sala de espera. Al entrar, la cliente se mostró muy molesta por la impuntualidad de la terapeuta (regla 2). Se utilizó este malestar para trabajar la empatía:

T: "¿Qué habrías hecho tú si estuvieras atendiendo a otras personas que no se marchaban? Intentando intercalar los marcos relacionales deícticos yo-tú.

C: "No lo sé" (CCR1).

Se interpretó que estaba evitando dar una respuesta con la función de demostrar que estaba enfadada. Así, se extinguió la CCR1 con intención de volver a hacerle la misma pregunta más adelante.

T: "¿Crees que he tardado con la intención de molestarte?"

C: "No lo sé" (CCR1) Se extinguió la CCR1.

T: "Ayúdame a mejorar esto, para que pueda hacerlo mejor la próxima vez que me ocurra."

C: "Puedes ponerte una alarma. O decir ya es la hora. Tu hora (de sesión) ha terminado" (CCR2).
T: "iPues eso sería una buena idea! gracias por tus consejos! Intentaré llevarlos a cabo la próxima vez que me ocurra."

T: "¿En casa te molestas así, con tu familia?". Se intentó comparar dentro-fuera de la sesión, según los marcos deícticos espaciales aquí-allí.

C: Silencio (CCR1).

$T:$ "¿Te dura tanto el enfado normalmente?"

C: Silencio (CCR1).

T: "Qué crees que puedo hacer para que dejes de estar enfadada conmigo?

C: No hay respuesta, pero sonríe (CCR1).

T: "Estás sonriendo, así que me haces pensar que no estás enfadada, porque las personas enfadadas normalmente no le sonríen a la persona con quien están enfadadas. Me estás haciendo un lío: ¿estás enfadada conmigo?"

C:"Tú sabrás" (CCR1).

T:"No lo sé porque no me lo dices. No me dejas seguir la sesión, porque minas la conversación... no puedo hablar contigo así si no me respondes."

$C$ : Después de permanecer en silencio, dijo "Estás perdiendo que sea de tu equipo."

No se siguió insistiendo, ya que se pensó que se estaba reforzando las negativas. Se decidió cambiar de conversación y preguntar por su abuela y su hermana. Se obtuvo una respuesta por parte de la cliente, que fue reforzada. Al final de la sesión, tras un comentario de Teresa, volvió a trabajarse la empatía en la misma línea que al principio:

C: "No he podido escribirte el correo que me pediste, he tenido que hacer otras cosas..."

T: "No te preocupes. No me enfado por eso. Entiendo que tenías cosas que hacer y no has podido escribirme. Si yo me enfadara por eso, ¿tú como te sentirías?" 
C: "Me sentiría mal" (CCR2).

T: "¿Y cómo crees que me siento yo ahora que tú te has enfadado porque yo he sido impuntual?"

\section{C: "Te sientes mal" (CCR2).}

Entre las sesiones 11 y 12, la cliente envió un correo electrónico de manera espontánea a la terapeuta pidiendo disculpas por su actitud en consulta y por haberla hecho sentir mal (CCR2). Para reforzar esta mejoría, se le envió de manera inmediata una respuesta aceptando las disculpas y agradeciendo el mensaje.

En la sesión $11^{a}$, Teresa describió que con su hermana y con su abuela apenas aparecían problemas en casa y que su relación había mejorado. Al comentar lo sucedido durante la última sesión, apareció una respuesta "yo X": "Me siento destrozada por cómo te dejé" (CCR2). Se mostró dispuesta a trabajar en cosas nuevas muy evitativas hasta ahora para ella, enfrentándose a la sensación de fracaso (CCR2): leer textos más largos, aprender las horas del reloj, escribir redacciones y corregirlas, etc., y no aparecieron negativas, ni quejas (CCR2).

Al comienzo de la sesión $12^{\text {a }}$ se le preguntó cómo iban las cosas en casa y contestó que "como siempre" (CCR1). Se le moldeó para que fuera sensible a los cambios que se habían estado produciendo desde que venía a consulta:

T: “CCómo está el ambiente en tu casa ahora?"

C: "Bien"

T: "¿Y antes, cómo era el ambiente en casa?"

C: "Era horrible"

T: "Entonces, ¿cómo van las cosas por casa ahora?"

\section{C: "Mucho mejor que antes."}

Se le expuso a Teresa que se había observado que había dado un manotazo a su madre en la cabeza en sala de espera (CCR1):

T: “¿Cómo crees que se siente ella cuando le haces eso?"

C: "No lo sé, pregúntaselo a ella. Ella sabrá cómo se siente" (CCR1)

T: "No te pregunto cómo se siente, sino cómo crees tú que ella se siente"

C: "Mal" (CCR2)

T: "Claro, yo también me sentiría mal si me golpearas. ¿Cómo te sentirías tú si alguien te lo hiciera a ti?"

\section{C: "Mal. Me enfadaría."}

Se le preguntó qué cambios veía en ella desde que venía a tratamiento, lo que provocó una serie de quejas (CCR1): "Estas preguntas son muy difíciles", una falta de respeto hacia la terapeuta ("iqué pesada eres!"), risas histriónicas y silencios.

T: "¿Qué te hace reír?"

C: "Es que no avanzamos ni hacia atrás ni hacia delante" (refiriéndose a la sesión de hoy).

T: “¿Cuál crees que sería la solución para poder avanzar?"

Esta pregunta provocó un incremento las CCR1.

T: "OK, ya veo que no quieres hablar sobre esto. No lo haremos. ¿Tú qué quieres que hagamos?"

Después de un silencio de menor duración que los que aparecían en situaciones similares, la cliente respondió "Bueno, ¿qué quieres que te diga sobre mi?". Fue la primera vez que después de ofrecer hacer lo que ella propusiera, se mostró dispuesta a hablar de ella misma, situación que antes había evita- 
do, sin requerírselo (CCR2). Se incrementaron las respuestas del tipo "yo X": respondió que antes era agresiva y la gente no quería estar cerca de ella (CCR3). Cuando se le preguntó cómo era ahora, dijo: “Ahora soy normal... no sé... no me lo había planteado nunca; ¿tú cómo me ves?". Para evitar reforzar un control público de su yo, se extinguió ésta pregunta. Se le pidió que intentara verse a ella misma a través de una pantalla de cine. Tras este ejercicio, comentó: "Ahora soy cariñosa, me pones en duda, nunca había pensado en esto..." eliminando así la dependencia a la opinión de la terapeuta (CCR2). Se trabajó con un ejercicio de auto-observación para mejorar las relaciones "yo X" y ponerlas bajo control privado, tal y como proponen Valero y Ferro (2015). Cuando se le volvió a preguntar cómo era ella aquí y ahora, no se obtuvo respuesta y se le dieron ayudas:

T: “¿Eres generosa o egoísta?"

C: "Las dos cosas"

T: "Aquí eres generosa. Por ejemplo, un día me trajiste un trozo de pastel que habías hecho en casa. ¿Crees que en casa eres generosa?"

C: "Si, en casa si"

T: "¿Estas alegre o triste?"

C: "Depende de cómo me levante"

Durante esta sesión, aceptó sistemáticamente las correcciones de su lenguaje (CCR2) y al final de ésta, se mostró colaboradora en sesión, aceptando realizar actividades de lectura y escritura (CCR2). Se jugó a un juego que ella eligió de manera espontánea (CCR2) y aceptó el fracaso (perdió) (CCR2), dando la enhorabuena espontáneamente (CCR2) a la terapeuta cuando ésta ganó.

\section{Resultados}

Este trabajo describe cómo se ha producido un cambio a lo largo del proceso terapéutico y una mejora en las relaciones terapéuticas y en las de intimidad de la cliente. En la sesión 2 , se mostró desconfiada hacia la terapeuta, tenía una actitud no colaboradora y fueron numerosas las quejas y las evitaciones (CCR1). En la sesión 3 comenzaron a aparecer CCR2. Durante la sesión 4 no hubo mejorías. Este hecho pudo deberse a que en dicha sesión se trabajó en la corrección del lenguaje de una manera artificial a través de ensayos de imitación vocal. Una hipótesis que se planteó fue su grado de aversión hacia esta tarea y que las tareas de lecto-escritura podría considerarlas infantiles para ella. Un objetivo prioritario fue su mejora en el habla, ya que a menudo lo que decía resultaba ininteligible, y se intentó trabajar en las primeras sesiones, pero fue un obstáculo para conseguir una buena relación terapéutica. Teniendo en cuenta que cuando hay demasiadas CCR1 durante la sesión es posible que el cliente abandone la terapia prematuramente (Tsai \& Reed, 2012), se decidió que a partir de la sesión 5 se trabajaría en el lenguaje de una manera más natural y espontánea, con auto-revelaciones de la propia terapeuta como consecuencias y marcando como objetivo conseguir una relación terapéutica autentica y genuina, como proponen los autores de FAP (Kohlenberg \& Tsai, 1991; Tsai et al., 2009). La sesión 5 podría ser considerada un punto de inflexión en el tratamiento, ya que hay un cambio de actitud hacia la terapeuta, mostrándose la cliente más colaboradora y flexible. En la sesión 6 habló por primera vez de su pasado y 
apareció una relación "yo X" de manera espontánea. A partir de esta sesión la familia informó que se estaban generalizando las mejorías en casa. La empatía se consideró un objetivo clave a partir de las sesiones 7 y 8 en adelante. El trabajo se centró en las relaciones "yo X" (con distintos marcos deícticos: yo-tú, yo aquí-yo allí, yo entonces-yo ahora). Fue en la sesión 9 cuando aparecieron los primeros comentarios de la cliente poniéndose en lugar de otros y atribuyéndose parte de responsabilidad en disputas familiares. En la sesión 10, se hizo esperar en la sala de espera a la cliente, lo que evocó una actitud inflexible y un incremento de CCR1, que fueron trabajadas con distintos marcos deícticos. En la sesión 11 la cliente se mostró más colaboradora y mostró una relación "yo X" de forma espontánea, por lo que se podría concluir que el bloqueo de CCR1 en la sesión anterior había sido efectivo. En la sesión 12 Teresa, de forma espontánea, estuvo dispuesta a hablar sobre ella misma. Se moldeó que se hablara sobre los cambios producidos durante el desarrollo de las sesiones, y sobre ella misma con un repertorio más amplio. De forma general, la corrección de la lecto-escritura se fue trabajando gradualmente a final de cada sesión a partir de la 9a, adaptando la tarea al repertorio de la cliente.

La intervención recogida en este trabajo corresponde a la fase inicial e intermedia del tratamiento con FAP, y actualmente Teresa continúa en terapia. Se sigue trabajando para eliminar las CCR1 y producir un aumento de las CCR2 y CCR3. Cada vez son menos frecuentes las situaciones de ira que la cliente presenta tanto dentro como fuera de la sesión. Ahora utiliza más mandos directos y elaborados de forma asertiva. En sesión, se está enfrentando a hablar de su pasado revelando cada vez más información. Acepta las correcciones, tanto en el lenguaje, como en tareas de lectura y escritura, lo que ha dado lugar a integrar en la terapia nuevas tareas (aprender a usar internet y el móvil, el manejo del dinero, la escritura de relatos cortos, el cálculo mental, etc.). Concretamente en el área del lenguaje, se corrige sin necesidad de ayudas cuando se percata de que no ha pronunciado bien una palabra o no ha elaborado correctamente una frase. La relación terapéutica ha mejorado, y se dan muestras de afecto por parte de la cliente. Estos repertorios se han generalizado al área familiar y social de Teresa, y su familia así lo reconoce. Está participando en diferentes talleres de manualidades y planea iniciar un curso de cocina, aún sabiendo que éste le exigirá realizar además tareas de lecto-escritura. Podemos decir que a día de hoy Teresa disfruta de mejores relaciones de intimidad y sociales y éstas han permitido que se deje ayudar para mejorar otras áreas que eran deficitarias.

\section{Discusión}

A la vista de estos resultados, se podría decir que la FAP está siendo efectiva para tratar a una persona con discapacidad intelectual, con una sospechada historia de maltrato y con problemas en sus relaciones de intimidad. También se puede hipotetizar que un problema del yo moderado en forma de un yo inestable, estaba en la base de esos problemas en las relaciones de intimidad, en la línea expuesta por Valero y Ferro (2015). 
La aparición de publicaciones e investigaciones sobre FAP se ha multiplicado en los últimos años (ver: Valero \& Ferro, 2015 y Ferro, Valero, \& López, 2016). La FAP se considera un marco terapéutico efectivo en casos donde se presentan problemas en las relaciones de intimidad. En el caso expuesto parece que la intervención desde FAP está consiguiendo eliminar los problemas de relación de la cliente, su hostilidad y su agresividad ante la terapeuta. Al principio de la intervención, las CCR1 aparecían en cada sesión con mucha frecuencia, y se hipotetizó que el tipo de problemática que presentaba podría estar relacionado con una ineficacia en sus relaciones de intimidad. La terapeuta bloqueó las CCR1 aunque no de manera sistemática para no generar un estado aversivo que pueda provocar el abandono de la terapia. A pesar de esto, y ante un posible castigo excesivo para la cliente, la terapeuta cambió la estrategia para convertir la situación en positivamente reforzante. La discapacidad intelectual leve no es relevante en este caso, ya que no impide un trabajo desde FAP creando un contexto terapéutico genuino y auténtico entre ambas personas en terapia. La posible historia de maltrato recibido y el abandono que vivió la cliente podría ser una hipótesis de la génesis de los problemas del yo que presentaba y como consecuencia, los problemas en las relaciones de intimidad. Uno de los objetivos terapéuticos fue conseguir la experiencia del yo como un contexto (la toma de perspectiva mencionada) y esta experiencia se consigue a través del yo como un proceso en la propia terapia, realizando ejercicios de auto-observación y tareas en la sesión clínica a través de las relaciones deícticas expuestas. Como se dijo, esa falta de empatía es un problema del marco interpersonal yo-tú que tiene que ver con esa toma de perspectiva, necesaria en la empatía. Y que está en la base de otros problemas psicopatológicos relacionados con estos marcos deícticos, como los problemas que se dan en los Trastornos Disociales, en algunos Trastornos de la Personalidad (Narcisismo, Límite), e incluso, en el autismo de alto funcionamiento. Desde FAP, se recomienda comparar lo que ocurre dentro-fuera, a través de los otros marcos deícticos (aquí-allí y antes-ahora) con el objetivo de conseguir la transferencia tanto de los problemas como de los resultados que se dan en el proceso de cambio terapéutico.

En general, desde FAP el tratamiento de los problemas del yo radica en transferir el control de las respuestas "yo X" de lo público a lo privado. La recomendación más general es el reforzamiento de cualquier expresión en ausencia de claves o indicaciones externas específicas. El tratamiento consiste en fortalecer todas las unidades "yo X" bajo control privado, lo cual ayudará también a la emergencia final del control privado sobre el yo, como se ha expuesto en este caso y anteriormente (Ferro, López \& Valero, 2012).

El moldeamiento de la terapeuta desde FAP parece que está siendo efectivo en un caso en el que otras intervenciones más clásicas y menos naturales habían fracasado. La adaptación a los repertorios y habilidades de la cliente en cada momento en este trabajo y los ejemplos en él expuestos han permitido trabajar en otras áreas en las que la evitación de la cliente lo impedía. En ningún momento se intervino 
directamente con la familia, dando instrucciones de lo que debía de hacer, y que como se muestra, no fue necesario.

Los autores son conscientes de las limitaciones que tiene este estudio de caso en el que no hay medidas previas ni posteriores al tratamiento, ni se ha usado un diseño experimental que demuestre de forma válida la efectividad de la intervención. Desde un punto de vista cuantitativo este caso clínico no posee datos. Pero de acuerdo con Valero y Ferro (2015), la medida de la efectividad de una intervención no solo está basada en los datos pre-post, sino también y no menos importante, en la utilidad terapéutica de lo que se describe, dando validez clínica y ecológica a los resultados. $\mathrm{Ni}$ que decir tiene, que el uso de diseños experimentales de sujeto único más potentes (diseños de línea base múltiple a través de sujetos, situaciones, etc.) reduciría esta limitación. También se reconoce la necesidad de replicación de la intervención en otras personas que muestren esta problemática. Aunque también somos conscientes de la dificultad de encontrar muestras de clientes equivalentes con esas reacciones de hostilidad que permitan esta investigación.

La importancia del presente trabajo radica en varias cuestiones. En primer lugar, en la descripción de las interacciones terapeuta-cliente, como un ejemplo de intervención en un caso de discapacidad leve, la mejora de su yo y sobre todo, en la relación terapéutica y en las relaciones de intimidad desde FAP. En un segundo lugar, la novedad del mismo es exponer la problemática de una cliente hostil ante el terapeuta y el moldeamiento que realiza la tera- peuta desde un marco de intervención desde la FAP. Finalmente, tiene una implicación para la práctica profesional, como un modelo para mejorar la relaciones terapéuticas difíciles en psicoterapia.

\section{Referencias}

Asociación Americana de Psiquiatría (2013). Guía de consulta de los criterios diagnósticos del DSM 5. Arlington, VA, Asociación Americana de Psiquiatría.

Barnes-Holmes, D., Hayes, S.C., \& Dymond, S. (2001). Self and Self-Directed Rules. En S.C. Hayes, D. Barnes-Holmes \& B. Roche (eds.) Relational Frame Theory. A post-Skinnerian Account of Human Language and Cognition. (pp.: 119-139). New York: Kluwer Academic.

Dymond, S. \& Roche, B. (2013). Advances in Relational Frame Theory. Research and Application. Oakland: New Harbinger.

Ferro-García, R., López-Bermúdez, M. A., \& Valero-Aguayo, L. (2012) Treatment of a disorder of self through Functional Analytic Psychotherapy. Internacional Journal of Behavioral Consultation and Therapy. 7, 2-3, 45-51. http://dx.doi.org/10.1037/h0100936 Recuperado de https://psycnet.apa.org/ record/2012-22649-008

Ferro-García, R., Valero-Aguayo, L., \& LópezBermúdez,M.A. (2009).Laconceptualización de casos clínicos desde la psicoterapia analítica funcional. Papeles del Psicólogo, 30, 3, 255-264. Recuperado de https://www. redalyc.org/pdf/778/77811790009.pdf

Ferro-García, R., Valero-Aguayo, L., \& LópezBermúdez, M.A. (2016). Fundamentos, características y eficacia de la Psicoterapia Analítica Funcional. Análisis y Modificación de Conducta. 41, 163-164, 1-28. http://hdl.handle. net/10272/12310 Recuperado de http:// rabida.uhu.es/dspace/handle/10272/12310

Hayes, S.C.,Barnes-Holmes, D.,\&Roche,B.(2001). Relational Frame Theory. A post-Skinnerian Account of Human Language and Cognition. New York: Kluwer Academic. https://doi. org/10.1348/000712604773952467 
Kanter, J.W., Weeks, C.E., Bonow, J.T., Landes, S.J., Callaghan, G.M., \& Follette, W.C. (2009). Assessment and case conceptualization. En M. Tsai, R.J. Kohlenberg, J.W. Kanter, B. Kohlenberg, W.C. Follette, \& G.M. Callaghan (Eds.): A guide to functional Analytic Psychotherapy. Awareness, courage, love and behaviorism (pp. 37-59). New York: Springer. https://doi.org/10.1007/978-0387-09787-9_3

Kohlenberg, R.J. \& Tsai, M. (1991/2008). Functional Analytic Psychotherapy. Creating intense and curative therapeutic relationship. New York: Plenum Press. (Traducción Servicio de Publicaciones Universidad de Málaga).

Kohlenberg, R. J. \& Tsai, M. (1998). Healing Interpersonal Trauma with the Intimacy of the Therapeutic Relationship. En V.M. Follette; J.I. Ruzeg, \& F.R. Abueg (Eds.). Cognitive-Behavioral Therapies for Trauma. (pp.: 305-320). New York: Guilford.

Kohlenberg, R.J. \& Tsai, M. (2000). Radical behavioral help for Katrina. Cognitive and Behavioral Practice, 7(4), 500505. https://doi.org/10.1016/S1077-

7229(00)80065-6 Recuperado de https:// www.sciencedirect.com/science/article/pii/ S1077722900800656

Kohlenberg, R.J., Tsai, M., Ferro-García, R., ValeroAguayo, L., Fernández-Parra, A., \&Virués-Ortega, J. (2005). Psicoterapia analítico-funcional y terapia de aceptación y compromiso: Teoría, aplicaciones y continuidad con el análisis del comportamiento. International Journal of Clinical and Health Psychology, 5(2), 349-371 Recuperado de https://www.redalyc.org/ html/337/33750209/

Kohlenberg, R. J., Tsai, M., Kanter, J. W., \& Parker, C. R. (2009). Self and Mindfulness.

En M. Tsai, R. J. Kohlenberg, J. W. Kanter, B. Kohlenberg, W.C. Follette, \& G. M

Callaghan, G.M. (Eds). A Guide to Functional Analytic Psychotherapy. Awareness, Courage, Love and Behaviorism. (pp.: 103130). New York: Springer. https://doi. org/10.1007/978-0-387-09787-9

Kohlenberg, R.J., Kanter, J.W., Bolling, M.Y., Parker, C.R., \& Tsai, M. (2002). Enhancing Cognitive Therapy for depression with
Functional Analytic Psychotherapy: Treatment guidelines and empirical findings. Cognitive and Behavioral Practice, 9(3), 213-229. https://doi.org/10.1016/ S1077-7229(02)80051-7 Recuperado de https://www.sciencedirect.com/science/ article/pii/S1077722902800517

Martín-Murcia, F. \& Ferro García, R. (2015). Fenomenología y psicopatología en las terapias de tercera generación. Psychology, Society \& Education, 7(1), 97-106. https://doi.org/10.25115/psye.v7i1.543 Recuperado de http://ojs.ual.es/ojs/ index.php/psye/article/view/543

McHugh, L., Stewart, I., \& Hooper, N. (2012). a Contemporary Functional Analytic Account of Perspective Taking. En L. McHugh, \& I. Stewart (eds.) The Self and Perspective Taking. Contributions and Applications from Modern Behavioral Science. (pp.: 55-71). Reno: Context Press.

Pérez Álvarez, M. (2014). Las terapias de tercera generación como terapias contextuales. Madrid: Ed. Síntesis.

Skinner, B.F. (1953/1970). Science and human behavior. New York: The Macmillan Company. (Traducción al español Ed. Fontanella)

Skinner, B.F. (1957/1981). Verbal behavior. Acton, MA: Copley Publishing. (Traducción al español Ed. Trillas).

Tsai, M., Kohlenberg, R.J., Kanter, J.W., \& Waltz, J. (2009). Therapeutic Technique: The Five Rules. En M. Tsai, R. J. Kohlenberg, J. W. Kanter, B. Kohlenberg, W.C. Follette, \& G.M. Callaghan (Eds). A Guide to Functional Analytic Psychotherapy. Awareness, Courage, Love, and Behaviorism. (pp.: 61-102). New York: Springer. https://doi.org/10.1007/9780-387-09787-9

Tsai, M., Plummer, M.D., Kanter, J.W., Newring, R.W., \& Kohlenberg, R. J. (2010). Therapist griefand Functional Analytic Psychotherapy: Strategic self-disclosure of personal loss. Journal of Contemporary Psychotherapy, 40(1), 1-10. https://doi.org/10.1007/ s10879-009-9116-6 Recuperado de https:// link.springer.com/article/10.1007/s10879009-9116-6 
Tsai, M., \& Reed, R. (2012). Working in-vivo with client sense of unlovability. International Journal of Behavioral Consultation and Therapy, 7(2-3), 147-150. http:// dx.doi.org/10.1037/h0100950 Recuperado de https://psycnet.apa.org/ record/2012-22649-022

Valero, L. \& Ferro, R. (2015). Psicoterapia Analítica Funcional. El análisis funcional en la sesión clínica. Madrid: Ed. Síntesis.

Valero, L. \& Ferro, R. (2017). Tratando mediante la Psicoterapia Analítica Funcional. Madrid: Ed. Pirámide 